JPE 12-4-16

\title{
Phase Shift Control for Series Active Voltage Quality Regulators
}

\author{
Guochun Xiao ${ }^{\dagger}$, Guofei Teng*, Beihai Chen ${ }^{*}$, and Jixu Zhang* \\ ${ }^{\dagger *}$ School of Electrical Engineering, State Key Laboratory of Electrical Insulation and Power Equipment, \\ Xi’an Jiaotong University, Xi'an, China
}

\begin{abstract}
A phase shift algorithm based on the closed-loop control of dc-link voltage implemented on a series active voltage quality regulator (AVQR) is proposed in this paper. To avoid pumping-up the dc-link voltage, a general phase shift compensation strategy is applied. The relationships among the operation variables are discussed in detail, which is very important for guiding the design of both the main circuit and the control system. Then on the basis of an investigation of the dc-link voltage pumping-up from viewpoint of the active power flow, a novel phase shift control method based on the closed-loop of the dc-link voltage is proposed. This method can adjust the phase of the output voltage gradually and automatically according to the dc-link voltage variation without introducing a phase jump. The effectiveness of the proposed strategy is verified through simulations of a single-phase 5kVA prototype and laboratory experiments on both a single-phase 5kVA and a three-phase 15kVA prototype.
\end{abstract}

Key words: DC-link voltage pumping-up, Overvoltage, Phase shift control, Power quality, voltage quality regulator

\section{INTRODUCTION}

Among power quality problems, supply voltage disturbances are considered to be the most significant and critical, especially at the point of common coupling (PCC) [1], [2]. One effective approach that can help sensitive loads in low power distribution systems ride through such upstream disturbances is to employ a series active voltage quality regulator (AVQR). Generally, there are many different structures for an AVQR. One typical topology for a three-phase AVQR with a coupling transformer is shown in Fig. 1. The main circuit consists of a coupling transformer TB, a diode rectifier VD, a voltage-source inverter VT and an energy storage capacitor $\mathrm{C}$. Since the AVQR manages only the fluctuation part of the supply voltage, it has a smaller size and a lower cost than the other regulators that processes the full-load power [3]-[8].

The AVQR can be widely used in poor voltage quality distribution systems, such as the small networks in oil exploration areas and remote mining districts [8]. Since the

Manuscript received Oct. 19, 2011; revised May 25, 2012

Recommended for publication by Associate Editor Kyeon Hur.

†Corresponding Author: xgc@mail.xjtu.edu.cn

Tel: +86-29-82666243, Fax: +86-29-82665223, Xi'an Jiaotong Univ.

* School of Electrical Engineering, State Key Laboratory of Electrical Insulation and Power Equipment, Xi'an Jiaotong University, China

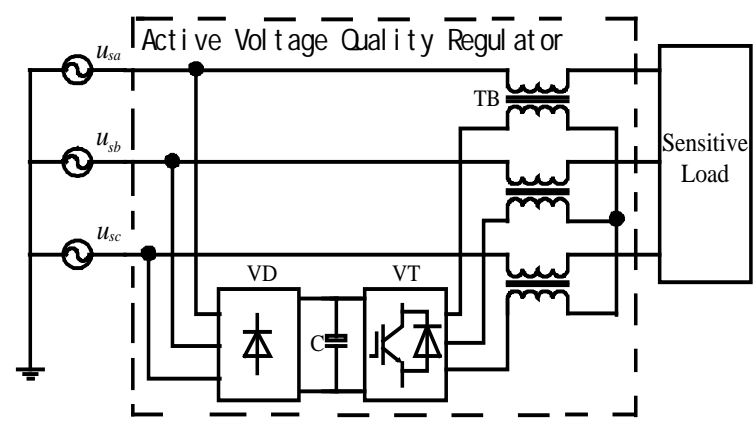

Fig. 1. Schematic of an AVQR with coupling transformer.

AVQR is on-line and able to mitigate many voltage quality problems, including long-term and short-term voltage disturbances in forms of overvoltages, undervoltages, voltage swells/sags, and the severely distorted voltage waveforms on sensitive loads. It is also able to maintain the output voltage at a desired magnitude and waveform. Dynamic voltage restorers (DVRs), on the other hand, are only suitable for off-line operation to effectively mitigate short-term voltage disturbances (sags/swells) [3]-[7].

Overvoltages are one of the major voltage quality problems in low power distribution systems. Voltage swells are generally the result of system faults, while overvoltages 
are not. They are generally caused by load variations on the system and system switching operations. Overvoltages can be the result of load switching (e.g., switching off a large load) or variations in the reactive compensation of the system (e.g., switching on a capacitor bank), or the states of a light load. This is especially true in residential and business buildings late at night. Poor system voltage regulation capabilities can cause overvoltages. Incorrect tap settings on transformers can also result in system overvoltages [9]. Overvoltages are mainly considered in this study as they are the main reasons for dc-link voltage pumping-up in AVQRs.

When a supply voltage is a long duration overvoltage, the phase of the compensation voltage will be opposite that of the supply voltage with the conventional in-phase injection scheme [10]. This will cause active power absorption of the regulator through the coupling transformer from the external power supply. Using a diode rectifier to charge the dc-link capacitor is an economical choice in the AVQR, but it cannot feed the energy back to the supply. Therefore, the active power absorption will lead to a dc-link voltage pumping-up of the capacitor within the regulator. Excessive and prolonged dc-link voltage pumping-up will increase the switching losses and lower the AVQR's efficiency, or even damage the storage and switching devices. Therefore, some measures have to be taken to avoid the dc-link voltage pumping-up in the AVQR.

Generally, there are three schemes for dealing with residual energy and to avoid dc-link voltage pumping-up, i.e. the energy-consumed, energy-feedback and phase shift control methods. All of them have strengths and drawbacks in application.

One cost-effective algorithm to avoid dc-link voltage pumping-up is through adjusting the phase of the injected voltage by adopting the zero-power injection strategy proposed in [11]-[14]. However, with the phase adjustment algorithm, both the phase displacement of the output voltage and the increase in the capacity necessary for the AVQR should be taken into consideration.

In fact, the implementation of zero-power injection will inevitably be accompanied by an initial phase jump of the output voltage [11], [12]. This type of sudden phase shift disturbance may interfere with the proper operations of the protected sensitive loads, such as adjustable speed drives and induction motors [15], [16]. Hence alleviation of the instantaneous phase jump has become an important issue [11], [12], [17]-[22].

In order to avoid the sudden phase jump of the output voltage, a progressive phase adjustment was considered through the different control algorithms proposed in [10]-[14], [17]-[20]. In [10]-[14], [20], the required phase shift angle needed to be calculated according to the supply voltage and the load condition in real time. This requires at least half or one system sampling cycle. This inevitably results in a slow response. In [17]-[19], the dc-link voltage of the energy storage device was used as a feedback signal to get the phase shift angle. Thus the phase jump of the output voltage can be avoided automatically. In [17], [18], the phase shift angle was generated by composing a phasor in phase with the supply voltage and another phasor in quadrature with a supply voltage whose magnitude was proportional to the dc-link voltage variation. Its implementation was very complicated. With the control strategy proposed in [19], minimum or zero power injection will be automatically achieved and the sudden phase jump of the output voltage can be avoided. However, in addition to its high computational expense, its implementation was also complex, and there were only simulation results provided.

Considering the shortcomings of the existing algorithms, a novel and simple phase shift method based on closed-loop control of the dc-link voltage implemented for an AVQR is proposed in the paper. With the proposed method, suppression of the dc-link voltage pumping-up and stabilization of the dc-link voltage can be successfully achieved. In addition, the progressive phase shift of the output voltage is automatically realized without introducing a sudden phase jump.

In addition, taking phase the shift algorithm in the AVQR will require a larger injected voltage than the conventional in-phase algorithms. With a larger injected voltage, the capacity of the inverter, the capacity of the isolation transformer, and the cost of the AVQR will increase. Unfortunately, papers discussing this issue elaborately are still difficult to find. Therefore, according to different supply and load conditions, reasonable and effective designs for both the main circuit and the control system of an AVQR are described in detail in the paper.

This paper begins with an analysis of phase shift control. Then the relationship between the supply overvoltage ratio, the load power factor, the required phase shift angle and the corresponding compensation voltage value are presented by a group of practical design curves, which may assist in the design of both the main circuit and the control system for low power distribution applications. Based on the relationship between the dc-link voltage and the active power flow, a novel closed-loop phase shift control method and its realization with a digital controller for a single-phase and a three-phase AVQR are proposed. A single-phase 5kVA and a three-phase $15 \mathrm{kVA}$ prototype have been developed to verify the proposed algorithm. Two AVQRs with ratings of 50kVA and $100 \mathrm{kVA}$ have been successfully integrated into a practical low power distribution system, and their effectiveness has been verified.

\section{Phase SHIFT CONTROL ANALYSIS}

Generally, both in-phase and phase shift compensation 
techniques can be used to control an AVQR. In-phase compensation means that the phase of the compensation voltage is the same as (undervoltage) or opposite (overvoltage) that of the supply voltage. Phase shift compensation means that there is a phase difference between the compensation voltage and the supply voltage. Consequently, there is a phase difference between the output voltage and the supply voltage.

\section{A. Phase shift control analysis for the inductive load}

As most of the loads in a power system are inductive, the phasor diagram under a voltage swell or overvoltage can be depicted as Fig. 2. Taking a single-phase system as an example, $\vec{U}_{S}, \vec{U}_{L}, \vec{U}_{C}, \vec{I}_{L}$ and $\varphi$ represent the supply voltage phasor, the output voltage phasor, the compensation voltage phasor, the load current phasor and the load power factor angle, respectively. The superscript $\hookrightarrow$ ' denotes the phasor variable and the symbol without this superscript denotes the RMS value [21], [22].

Using in-phase compensation, the magnitude of the compensation voltage is given as:

$$
U_{C}=U_{S}-U_{L}
$$

Assuming that $P_{S}$ and $P_{L}$ represent the output power and the power absorbed by the load, respectively, thus:

$$
\begin{aligned}
& P_{L}=U_{L} I_{L} \cos \varphi \\
& P_{S}=U_{S} I_{L} \cos \varphi
\end{aligned}
$$

Assuming that $\triangle P$ represents the active power injected into the AVQR, then from (2) and (3), the following can be derived:

$$
\Delta P=P_{S}-P_{L}=\left(U_{S}-U_{L}\right) I_{L} \cos \varphi
$$

It is known that the in-phase compensation scheme results in dc-link voltage pumping-up during voltage swells or overvoltages $\left(U_{S}>U_{L}\right)$.

The proposed phase shift technique is realized by adjusting the phase shift angle $\theta$ in the clockwise rotating direction as shown in Fig. 2. Supposing that $\vec{U}_{S}$ is a stationary reference phasor, $\vec{U}_{L}^{\prime}, \vec{I}_{L}^{\prime}$ and $\vec{U}_{C}^{\prime}$ represent the output voltage phasor, the load current phasor and the compensation voltage phasor after phase shift compensation, respectively. The magnitudes of $\vec{U}_{L}^{\prime}$ and $\vec{I}_{L}^{\prime}$ are equal to $\vec{U}_{L}$ and $\vec{I}_{L}$ for a fixed load, while the magnitude of the compensation voltage will be larger than that with the in-phase injection method. In this case, the load absorbing active power is still $P_{L}$, but the supply power can be expressed as:

$$
P_{S}=U_{S} I_{L} \cos (\varphi+\theta)
$$

The active power injected into the AVQR can be given as:

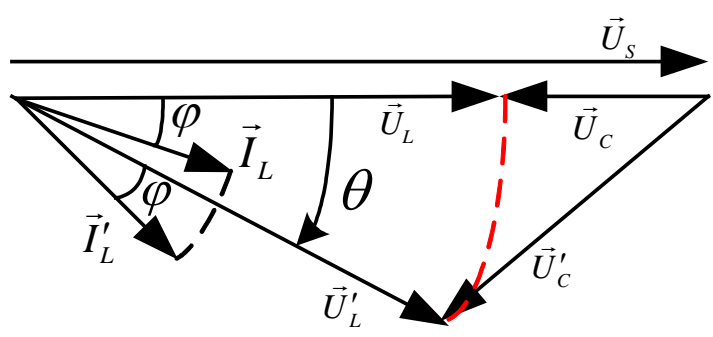

Fig. 2. Phasor diagram during overvoltage.

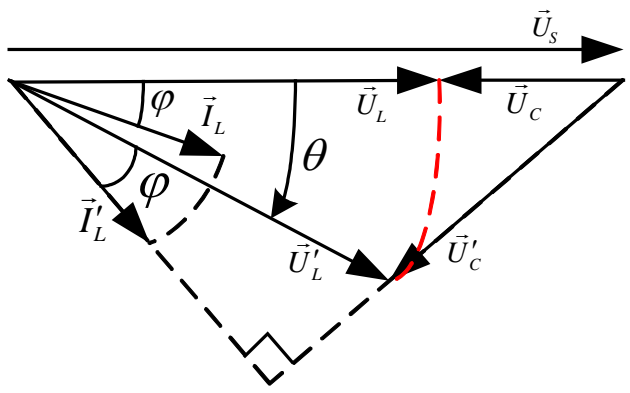

Fig. 3. No pumping-up for inductive load under

$$
\Delta P=P_{S}-P_{L}=I_{L}\left[U_{S} \cos (\varphi+\theta)-U_{L} \cos \varphi\right]
$$

The compensation voltage is obtained as:

$$
U_{C}^{\prime}=\sqrt{U_{S}^{2}+U_{L}^{2}-2 U_{S} U_{L} \cos \theta}
$$

Since $\theta>0$ with the phase shift compensation, thus:

$$
\sqrt{U_{S}^{2}+U_{L}^{2}-2 U_{S} U_{L} \cos \theta}>U_{S}-U_{L}
$$

Therefore:

$$
U_{C}^{\prime}>U_{C}
$$

One obvious advantage of the proposed scheme is that less active power is injected into the inverter. Consequently, the dc-link voltage pumping-up is suppressed. Especially, considering the possibility of operating at $\Delta P=0$ during voltage swells or overvoltages, from (6) there is:

$$
U_{S} \cos (\varphi+\theta)=U_{L} \cos \varphi
$$

In this case, as shown in Fig. 3, the load current $\vec{I}_{L}^{\prime}$ is perpendicular to the compensation voltage $\vec{U}_{C}^{\prime}$. Therefore, that no active power is injected into the inverter and the dc-link voltage no longer rises. The magnitude of the compensation voltage can be calculated as:

$$
\begin{gathered}
U_{C}^{\prime}=U_{S} \sin (\varphi+\theta)-U_{L} \sin \varphi \\
\text { phase shift compensation. }
\end{gathered}
$$

From (11), through mathematical derivations, without dc-link voltage pumping-up, the relationship between the compensation voltage, the supply voltage, the output voltage 
and the load power factor angle is given as:

$$
U_{C}^{\prime}=\sqrt{U_{S}^{2}-U_{L}^{2} \cos ^{2} \varphi}-U_{L} \sin \varphi
$$

The required phase shift angle can be derived as:

$$
\theta=\cos ^{-1}\left(\frac{U_{L}}{U_{S}} \cos \varphi\right)-\varphi
$$

Clearly, when $\varphi=0$, from (12) and (13), without introducing any dc-link voltage pumping-up, the required phase shift angle and the corresponding compensation voltage with a resistive load can be obtained:

$$
\begin{gathered}
U_{C}^{\prime}=\sqrt{U_{S}^{2}-U_{L}^{2}} \\
\theta=\cos ^{-1} \frac{U_{L}}{U_{S}}
\end{gathered}
$$

Since it is restricted by the maximum dc-link voltage provided by the AVQR, the maximum compensation voltage limit $U_{C \max }^{\prime}$ can also be determined as:

$$
U_{C}^{\prime}=\sqrt{U_{S}^{2}+U_{L}^{2}-2 U_{S} U_{L} \cos \theta}<U_{C \max }^{\prime}
$$

Thus, the maximum phase shift angle limit $\theta_{\max }$ under the AVQR's maximum compensation voltage is given as:

$$
\theta_{\max }=\arccos \left(\frac{U_{S}^{2}+U_{L}^{2}-U_{C \max }^{\prime 2}}{2 U_{S} U_{L}}\right)
$$

B. Compensation voltage and phase shift angle calculation

Under the proposed phase shift control, the phase shift angle $\theta$ and the compensation voltage $U_{C}^{\prime}$ are two important parameters. The phase shift angle $\theta$ and the compensation voltage $U_{C}^{\prime}$ are relative to the magnitude of the supply voltage and the load power factor. The overvoltage ratio $\delta$ of the supply voltage is defined as:

$$
\delta=\frac{U_{S}-U_{L}}{U_{L}} \times 100 \%
$$

From (12) and (13), assuming that $U_{L}=1$ p.u., the following equation can be obtained:

$$
\begin{gathered}
U_{C}^{\prime}=\sqrt{(1+\delta)^{2}-\cos ^{2} \varphi}-\sin \varphi \\
\theta=\cos ^{-1}\left(\frac{\cos \varphi}{1+\delta}\right)-\varphi
\end{gathered}
$$

When the overvoltage ratio $\delta$ is $5 \%, 10 \%, 15 \%, 20 \%, 25 \%$ and $30 \%$, respectively, and the load power factor is in the range of $0.5 \sim 1.0$, using (19) and (20), one can obtain the

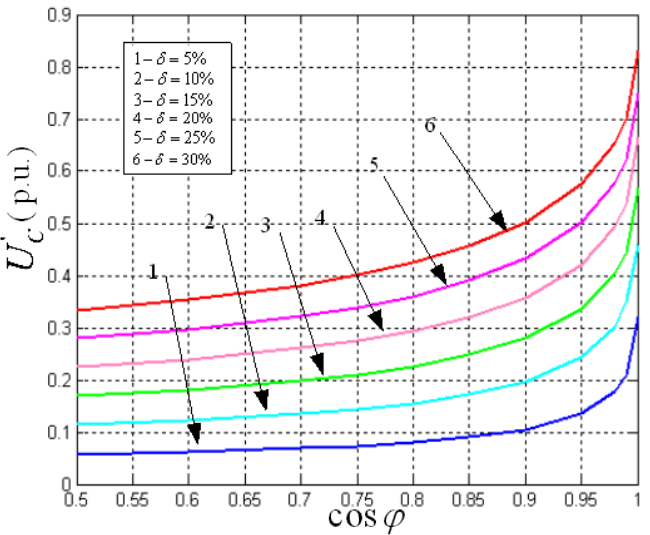

Fig. 4. Compensation voltage versus load power factor and overvoltage ratio.

curves of the compensation voltage $U_{C}^{\prime}$ versus the load power factor, as shown in Fig. 4, and the curves of the phase shift angle $\theta$ versus the load power factor, as shown in Fig. 5.

When the pumping-up of dc-link voltage does not exist, from Fig. 4 and Fig. 5, it can be seen that the higher the load power factor, the greater the need for compensation of the voltage and the phase shift angle. They both reach their maximum value when $\cos \varphi=1$ (pure resistive load). With the increase of the supply overvoltage ratio $\delta$, the need for compensation of the voltage and the phase shift angle will increase too.

From (19) and (20), with a pure resistive load (i.e. $\varphi=0$ ) and a given overvoltage ratio $\delta$, the maximum phase shift angle and compensation voltage without introducing a dc-link voltage pumping-up can be obtained as:

$$
\begin{aligned}
& U_{C}^{\prime}=\sqrt{(1+\delta)^{2}-1} \\
& \theta=\cos ^{-1}\left(\frac{1}{1+\delta}\right)
\end{aligned}
$$

Furthermore, from (21) and (22), the relationship between the overvoltage ratio $\delta$, the phase shift angle $\theta$ and the compensation voltage $U_{C}^{\prime}$ without any dc-link voltage pumping-up can be obtained, as shown in Fig. 6 and Fig. 7.

When deriving the aforementioned formulas, the losses of the inverter and the dc-link energy storage capacitors are not considered. With these losses (e.g., the switching loss), the practical compensation voltage and the phase shift angle without any dc-link voltage pumping-up will be smaller than the theoretical values when using the phase shift control. 


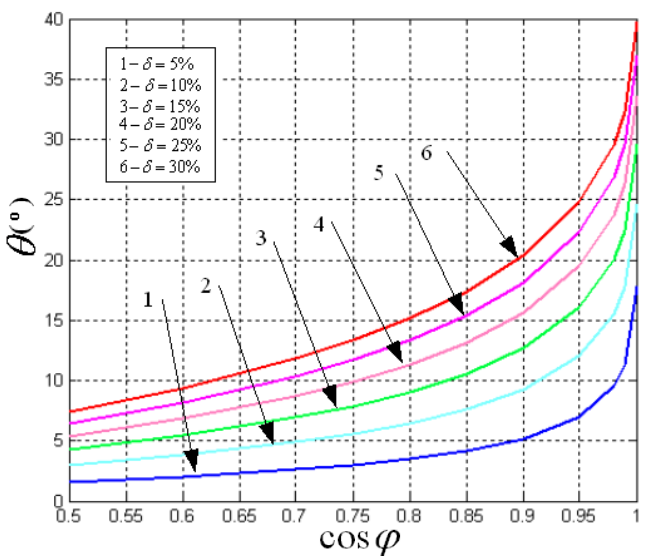

Fig. 5. Phase shift angle versus load power factor and overvoltage ratio.

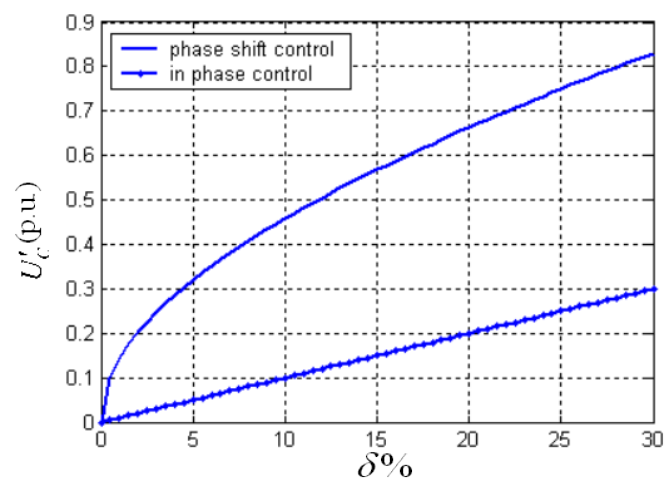

Fig. 6. Compensation voltage versus overvoltage ratio with pure resistive load (rated output voltage 1p.u.).

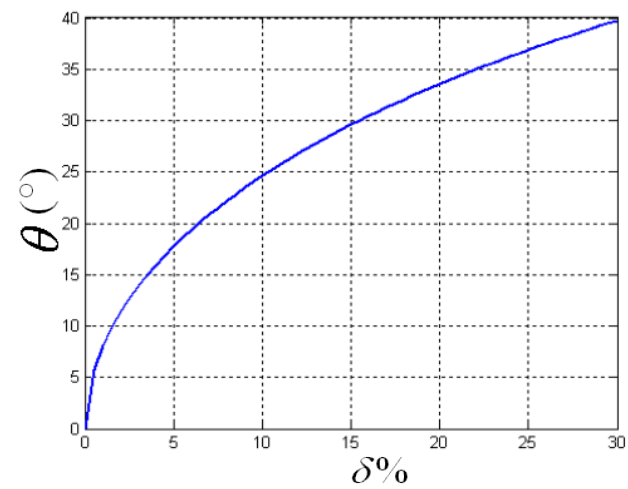

Fig. 7. Phase shift angle versus overvoltage ratio with pure resistive load.

\section{REAlization OF Phase Shift Control}

The phase shift control algorithm can suppress the dc-link voltage pumping-up effectively by decreasing the active power provided by the power supply under swells/overvoltages. However, how to realize the automatic regulation of the compensation voltage and the phase shift

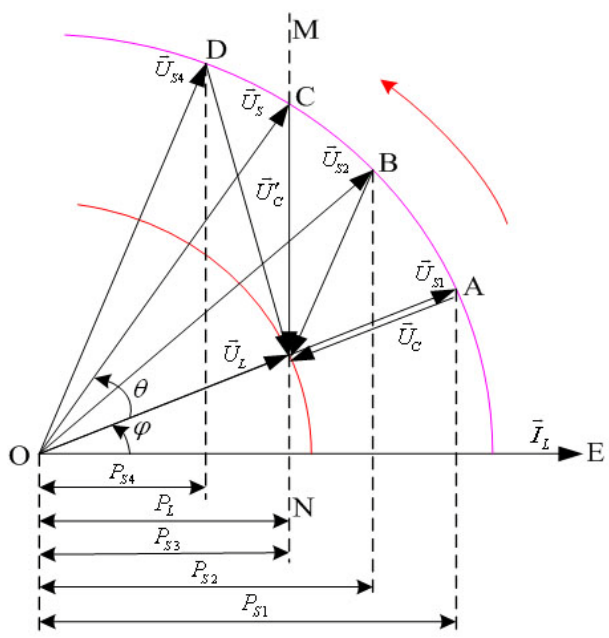

Fig. 8. Active power variation under phase shift control.

angle according to stochastic variations of the supply and the load still need to be discussed. In this section, a novel phase shift control strategy is proposed.

\section{A. Active power balance analysis}

Fig. 8 shows the principle of the phase shift automatic control, where the outer circle denotes the locus of the supply voltage $\vec{U}_{S}$ and the inner one denotes the locus of the output voltage $\vec{U}_{L}$. The inductive load is taken for to describe the realization of the phase shift regulation. Fig. 8 also shows the variation of the active power provided by the supply during the rotation of $\vec{U}_{S} \cdot P_{S 1} \sim P_{S 4}$ are the active powers provided by the supply under different conditions. $P_{L}$ is the active power absorbed by the load. All the other definitions are the same as in Fig. 2.

It should be pointed out that for a certain load (e.g., inductive load), the phase difference $\varphi$ between the output voltage $\vec{U}_{L}$ and the load current $\vec{I}_{L}$ is fixed. To make this simple, by taking $\vec{I}_{L}$ as the reference phasor, the phase of $\vec{U}_{L}$ is subsequently fixed. For the proposed AVQR, the magnitude and phase of the supply current are the same as those of the load current. Thus, it achieves the same effect when rotating $\vec{U}_{S}$ in one direction or rotating $\vec{U}_{L}$ in the reversed direction.

When $\vec{U}_{S}$ is located at point $A(\theta=0), \vec{U}_{L}$ is in phase with $\vec{U}_{S}$, assuming that $\vec{U}_{S}=\vec{U}_{S 1}$, which is referred to as in-phase compensation. The active power provided by the supply is $P_{S 1}$ and $P_{S 1}>P_{L}$ under overvoltages. As a result, the AVQR will absorb energy from the supply continuously 


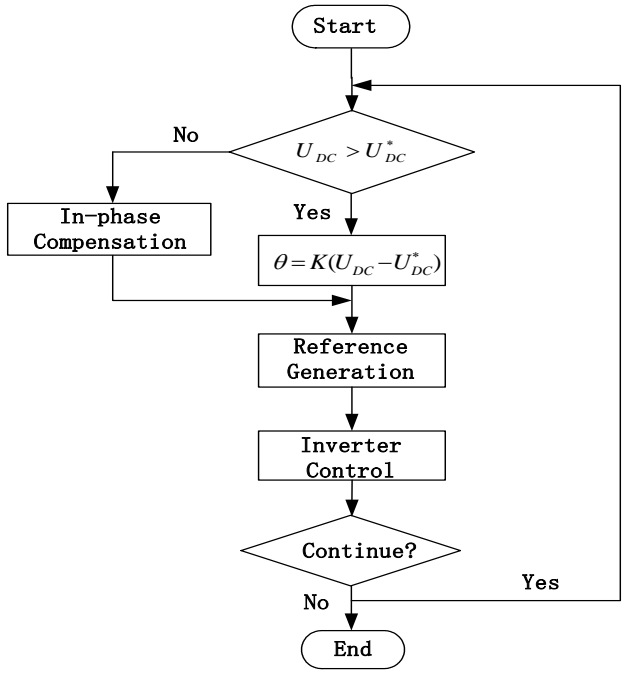

Fig. 9. Flow chart of phase shift control realized in a single-phase AVQR.

and the dc-link voltage pumping-up occurs subsequently. When $\vec{U}_{S}$ rotates to point $B$, assuming that $\vec{U}_{S}=\vec{U}_{S 2}$, the active power provided by the supply is reduced to $P_{S 2}$ and $P_{S 1}>P_{S 2}>P_{L}$, which means that the energy causing the dc-link voltage pumping-up decreases. When $\vec{U}_{S}$ rotates to points $C$ and $D$, the active power provided by the supply decreases to $P_{S 3}$ and $P_{S 4}$, respectively, and $P_{S 1}>P_{S 2}>P_{S 3}=P_{L}>P_{S 4}$. Thus the AVQR will release energy in the state of point $D$ and the dc-link voltage will decline accordingly. In theory, when $\vec{U}_{S}$ is located at point $C$, where $P_{S 3}=P_{L}$, the active power provided by the supply is equal to that supplied to the load, thus the dc-link voltage is maintained at a sustained value. In practice, when considering the losses of the AVQR, the active power provided by the supply should be a little larger than that supplied to the load to keep the dc-link voltage at a constant level.

Therefore, the variation of the dc-link voltage directly reflects the variation of the active power flow, and the active power flow can be controlled through the phase shift control algorithm. This means the dc-link voltage can also be regulated by the phase shift control algorithm. The dashed line $M N$ is the boundary line between the active power absorption and the release of the AVQR. When $\vec{U}_{S}$ rotates clockwise, the AVQR will absorb active power from the external system and cause the rise of the dc-link voltage. On the contrary, the AVQR releases the active power and results in a fall of the dc-link voltage. The quantity of the active power flow is dependent on the phase shift angle.

\section{B. Realization of the phase shift control for a single-phase} $A V Q R$

The flow chart for the phase shift control strategy realized in a single-phase AVQR is shown as in Fig. 9. The key issue is control of the dc-link voltage.

The first step is to set the reference value $U_{D C}^{*}$ as a threshold to enter the phase shift compensation state. Two factors should be taken into consideration when setting the threshold:

(1) It should be insured that the rectifier VD is out of operation during phase shift compensation, $U_{D C}^{*}$ should be higher than the natural rectifying voltage under the maximum overvoltage.

(2) The maximum compensation voltage provided by the AVQR should be ensured.

The values of the dc-link voltage are sampled, and the average value $U_{D C}$ should be calculated. Then the controller will make a comparison between $U_{D C}$ and $U_{D C}^{*}$. The AVQR will operate with in-phase compensation when $U_{D C}<U_{D C}^{*}$, otherwise it will automatically enter the phase shift control once the requirement $U_{D C}>U_{D C}^{*}$ is satisfied. The value of the phase shift angle is determined by:

$$
\theta=K\left(U_{D C}-U_{D C}^{*}\right)
$$

Here, $K$ is a coefficient to regulate the phase shift angle. The varying extent of the load phase is greatly affected by $K$, the load condition, and the dc-link capacitor's capacity.

The AVQR will continue to absorb energy from an external system as soon as the phase shift angle is insufficient. In addition, the dc-link voltage will rise under this condition. Consequently, the phase shift angle will increase, thus the energy absorbed by the AVQR will be gradually minimized. The dynamic regulation process will not end until the energy balance state is achieved. The dc-link voltage will become stable after this process, and the phase shift angle of the output voltage will achieve its minimum. As for an inductive load, the AVQR achieves zero active power transfer once the required shift angle, obtained from (13), is satisfied. In fact, considering the operating loss of the AVQR, the required phase shift angle will be slightly smaller than the theoretical value.

\section{Realization of the phase shift control for a three-phase $A V Q R$}

As for a three-phase AVQR, the required $120^{\circ}$ phase difference for each output voltage can only be ensured when the three phase voltages have the same phase shift angle. Otherwise, the normal operation of phase sensitive equipments will be disturbed. As mentioned in the above 
section, the choice of the phase shift angle mainly depends on the dc-link voltage. Thus, to achieve the same shift angle for the three phases, the same dc-link voltage value for the three phases should be ensured. However, this condition is difficult to satisfy. The following will result in a difference in the three-phase dc-link voltages:

(1) An imbalance in the three-phase supply voltage ;

(2) Differences among the three-phase loads;

(3) Manufacturing difference in the three-phase circuits.

Supposing that the phase shift control for each phase is still carried out according to the different dc-link voltage, an inconsistent phase shift angle and a violation of a $120^{\circ}$ phase difference rule by each phase will happen. Thus based on the realization of the phase shift control for a single-phase AVQR, a strategy for phase shift control for a three-phase AVQR is proposed, and the main steps are as follows:

(1) Find the maximum of the three-phase (phase $A, B, C$ ) dc-link voltage $U_{D C \max }$ :

$$
U_{D C \max }=\max \left(U_{D C A}, U_{D C B}, U_{D C C}\right)
$$

(2) Take $U_{D C \max }$ as the reference to carry out the phase shift compensation. The common phase shift angle of the three-phase AVQR is given by:

$$
\theta=K\left(U_{D C \max }-U_{D C}^{*}\right)
$$

(3) The following steps are the same as those shown in the single-phase case.

\section{SIMULATION AND EXPERIMENTAL VERIFICATION}

From Fig. 4, Fig. 6 and the analysis above, it can be seen that in order to suppress the dc-link pumping-up phenomenon, the necessary compensation voltage with phase shift control is much larger than that with in-phase control, and the higher the supply overvoltage ratio and the load power factor, the larger the necessary compensation voltage. An increase of the compensation voltage means to increase of the capacity of the inverter and the coupling transformer in the AVQR topology, as shown in Fig. 1, which is not cost-effective. Therefore, a single-phase configuration with an isolation transformer is proposed, as shown in Fig. 10. When the supply voltage is in undervoltage for a long time, the supply will inject energy into the dc-link of the AVQR through the rectifier. Accordingly, for the sake of electrical isolation, an isolation transformer $\mathrm{T}$ is necessary and its capacity is dependent on the undervoltage ratio.

Further investigations including both load-disturbance and supply-disturbance simulations and experiments for a single-phase 5kVA as well as a three-phase 15kVA prototype are carried out to verify the effectiveness of the proposed control strategy. The main parameters of the single-phase prototype are listed in Table I.

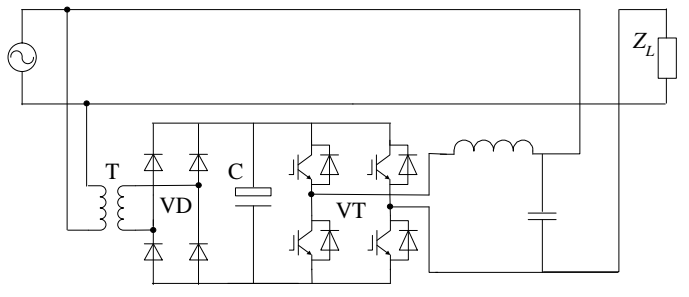

Fig. 10. Topology of a single-phase AVQR with an isolation transformer.

TABLE I

MAIN PARAMETERS

\begin{tabular}{cc}
\hline Items & Parameters \\
Prototype capacity & $5 \mathrm{kVA}$, single-phase \\
Isolation transformer $\mathrm{T}$ & $2 \mathrm{kVA}$, turns ratio 4:1 \\
Dc-link capacitor & $4700 \mu \mathrm{F} / 450 \mathrm{VDC}$ \\
Supply voltage & $180 \mathrm{~V}$ 260Vrms, 50Hz \\
Rated load voltage & $220 \mathrm{Vrms}$ \\
Load & $21 \Omega$ resistive load \\
Switching frequency & $15 \mathrm{kHz}$ \\
Dc-link threshold voltage & $145 \mathrm{~V}$ \\
Coefficient $K$ & 0.3 \\
\hline
\end{tabular}

\section{A. Simulation results}

Simulation results are presented in Figs.11 13 to verify the proposed phase shift control in the single-phase topology, as shown in Fig.10. In Fig.11, the steady-state simulation results are given under the condition that the supply voltage swells from $220 \mathrm{~V}$ to $240 \mathrm{~V}$, and the resistive load is connected to the system. In Fig.11, the phenomenon of phase shift is clearly illustrated in the waveform comparison between supply voltage and load voltage. The simulation results under different steady-state conditions are listed in Table II.

In Fig.12, the supply voltage is $240 \mathrm{~V}$ and the load is applied to the system at instant $0.3 \mathrm{~s}$. From the waveforms, it can be seen that the control system can maintain the load voltage at a rated value all the times. Moreover, the values of the DC-link voltage and the compensation voltage change according the analysis given above.

In Fig.13, the supply overvoltage occurs from $220 \mathrm{~V}$ to $240 \mathrm{~V}$ at instant $0.3 \mathrm{~s}$. The system can keep the load voltage at a constant value of $220 \mathrm{~V}$, which can effectively protect the sensitive loads in real systems. Similar to Fig.12, the process of phase shift control is well in accord with the theory analysis, which demonstrates the effectiveness of the proposed strategy.

\section{B. Single-phase experiment}

1) Steady-state experiment: The aim of the steady-state experiment is to verify the relationship among the supply overvoltage ratio, the required phase shift angle and the corresponding compensation voltage without introducing any 

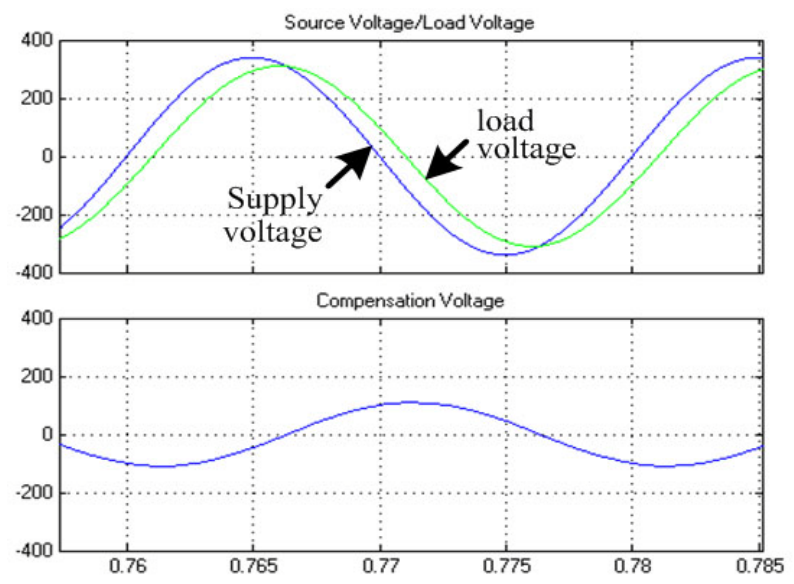

Fig. 11. Phase difference in steady-state.
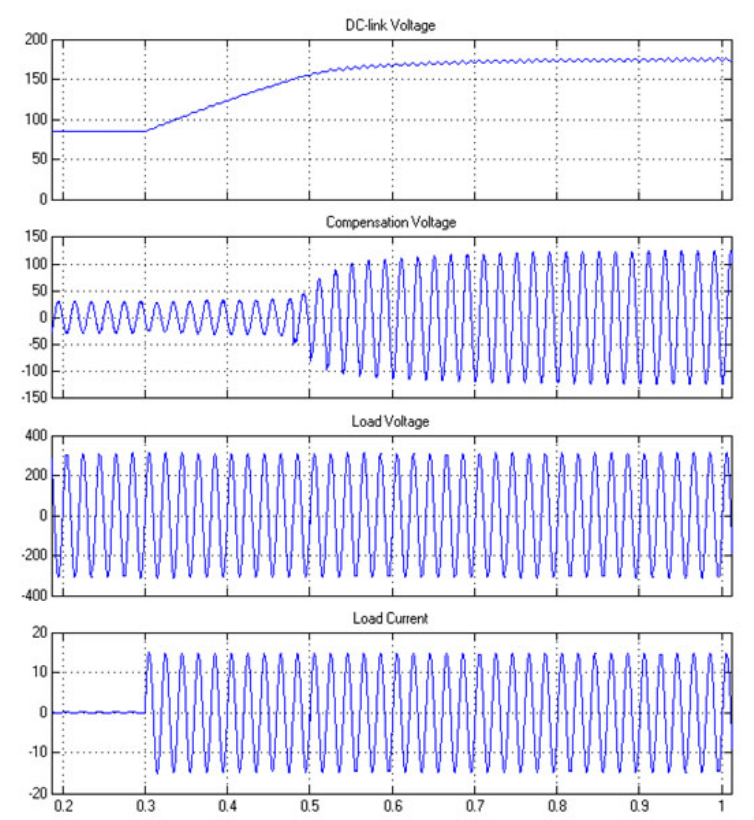

Fig. 12. Simulation result with load-disturbance.

dc-link pumping-up and verify the effectiveness of the theoretical analysis as well.

Table II lists the theoretical, simulation and experimental results of both the required phase shift angle and the corresponding compensation voltage with a $2 \mathrm{~kW}$ resistive load, while the supply overvoltage ratio is $5 \%, 10 \%$ and $14 \%$, respectively. The dc-link voltage with different overvoltage ratios is also shown in this table. The theoretical results of the necessary phase shift angle and the corresponding compensation voltage without introducing dc-link pumping-up are obtained by using (21) and (22).

From Table II, the experimental results are very close to both the theoretical results and simulation results, which indicates that the theoretical analysis is effective and can
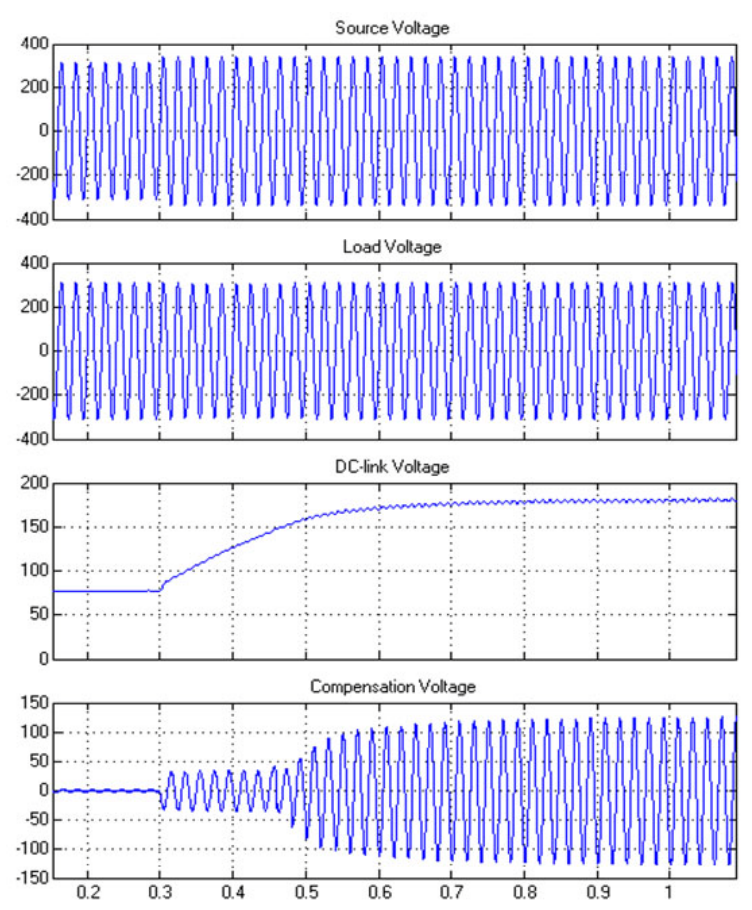

Fig. 13. Simulation result with supply-disturbance.

TABLE II

Single-PHASE STEAdY-STATE SimUlation AND EXPERIMENTAL RESULTS

\begin{tabular}{|c|c|c|c|c|}
\hline \multicolumn{2}{|c|}{ Overvoltage ratio } & $5 \%$ & $10 \%$ & $14 \%$ \\
\hline \multirow{2}{*}{$\begin{array}{c}\text { Compensation } \\
\text { voltage (V) }\end{array}$} & Theoretic & 70 & 101 & 121 \\
\cline { 2 - 5 } & Simulation & 59 & 91 & 114 \\
\cline { 2 - 5 } & Experiment & 48 & 90 & 112 \\
\hline \multirow{2}{*}{$\begin{array}{c}\text { Phase shift } \\
\text { angle (deg) }\end{array}$} & Theoretic & 17.8 & 24.6 & 28.8 \\
\cline { 2 - 5 } & Simulation & 14.4 & 21.6 & 27 \\
\cline { 2 - 5 } & Experiment & 12.6 & 19.8 & 25.2 \\
\hline $\begin{array}{c}\text { DC-link voltage } \\
(V)\end{array}$ & Simulation & 165 & 179 & 188 \\
\cline { 2 - 5 } & Experiment & 150 & 160 & 165 \\
\hline
\end{tabular}

guide the design of equipment. The experimental results are all slightly lower than the theoretical results. This is because the theoretical formulas do not take the losses of the inverter and the dc-link energy storage capacitors into consideration.

\section{2)Load-disturbance experiment}

Fig. 14 shows an outline of the load-disturbance experimental results, including the dc-link voltage (CH1), the compensation voltage $(\mathrm{CH} 2)$, the output voltage $(\mathrm{CH} 3)$ and the load current $(\mathrm{CH} 4)$ during the process of applying and removing the load, while the supply voltage is $242 \mathrm{~V}$ (i.e. the 
supply overvoltage ratio is 10\%). The output voltage is maintained at $220 \mathrm{~V}$ throughout the experiment. In Fig.14, the load is applied to the system at the instant of E, and removed at the instant of $\mathrm{H}$. From instant E, the inverter of the AVQR begins to absorb energy and the dc-link voltage experiences a nearly linear increase. The period of EF is the dc-link voltage pumping-up period. Once the dc-link voltage reaches the threshold value at instant $\mathrm{F}$, the phase shift control algorithm is automatically applied to replace the in-phase compensation method. A short dynamic phase shift process is experienced, during which the dc-link voltage do not continuously rise. However, the compensation voltage starts rising. Then, the system enters the steady-state phase shift compensation period and the dc-link voltage is maintained at a constant level. Nevertheless, the load is removed at the instant of $\mathrm{H}$. The dc-link voltage and the compensation voltage are decreasing gradually to the original state and the in-phase compensation method is automatically adopted again. It is remarkable that during the whole period, the output voltage is kept at the rated value without a sudden phase-jump.

Figs. 15 17 are partial enlarged views of Fig. 14. Fig. 15 is an enlarged view of period EF; Fig. 16 is an enlarged view at around instant F; and the enlarged view after removing the load is shown in Fig. 17.

From Fig.14 17, it can be seen that the effectiveness of restraining the pumping-up of the dc-link voltage, the progressive phase shift of the output voltage without introducing a phase-jump and the stabilization of the magnitude of the output voltage have been verified by the load-disturbance experiment.

It should be noted that the presented waveforms of the compensation voltage variation in the phase shift process are seldom found in other studies discussing phase shift control [10]-[13], [17]-[20].

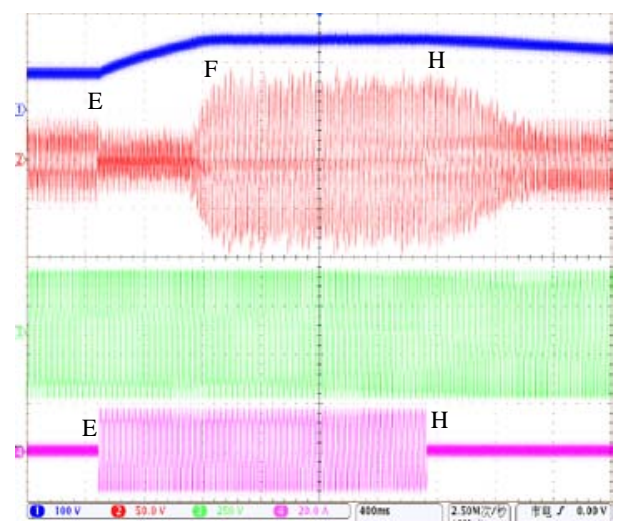

Fig. 14. Outline of the load-disturbance experiment.(CH1: dc-link voltage, $100 \mathrm{~V} / \mathrm{div}$; $\mathrm{CH} 2$ : compensation voltage, 50V/div; CH3: output voltage, 250V/div;CH4: load current, 20A/div; time axes: 400ms/div).

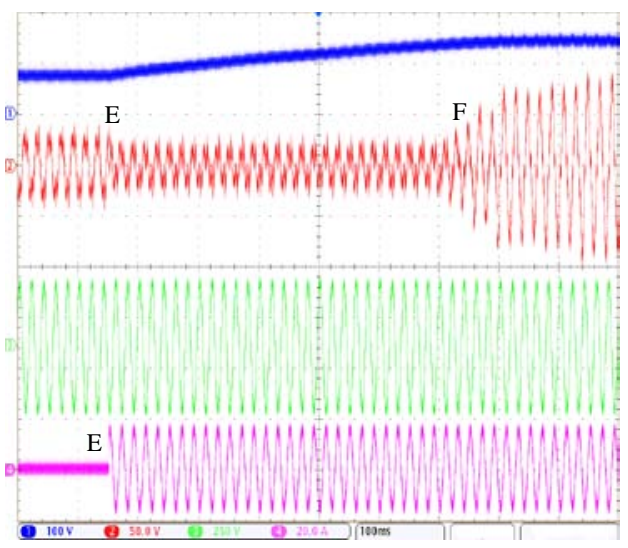

Fig. 15. Enlarged view of period EF. (CH1: dc-link voltage, 100V/div; CH2: compensation voltage, 50V/div; CH3: output voltage, 250V/div; CH4: load current, 20A/div; time axes: $100 \mathrm{~ms} / \mathrm{div})$.

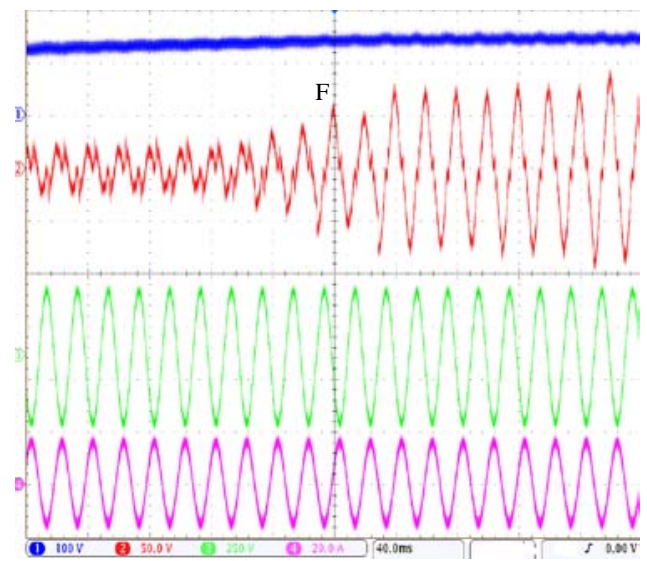

Fig. 16. Enlarged view around instant F. (CH1: dc-link voltage, 100V/div; CH2: compensation voltage, 50V/div; CH3: output voltage, 250V/div; CH4: load current, 20A/div; time axes: $40 \mathrm{~ms} / \mathrm{div})$.

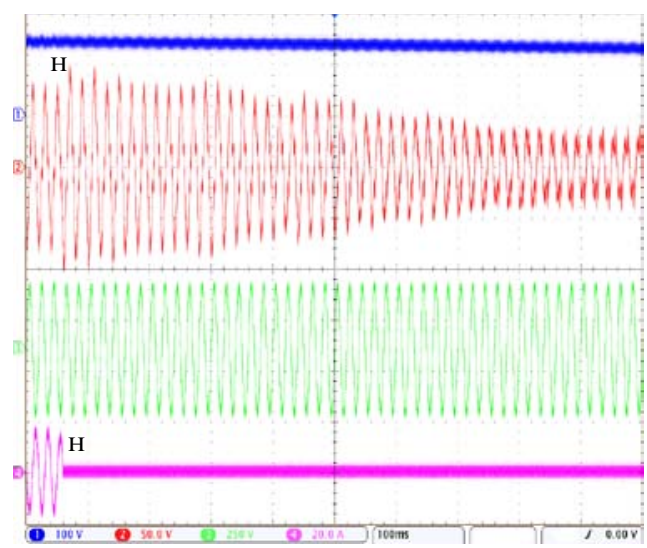

Fig. 17. Enlarged view after removing the load. (CH1: dc-link voltage, 100V/div; CH2: compensation voltage, 50V/div; CH3: output voltage, 250V/div; CH4: load current, 20A/div; time axes: $100 \mathrm{~ms} /$ div). 


\section{3)Supply-disturbance experiment}

Fig. 18 shows an outline of the supply-disturbance experimental results, including the supply voltage (CH1), the output voltage (CH2), the dc-link voltage ( $\mathrm{CH} 3)$ and the compensation voltage (CH4), when there are repeated supply voltage disturbances (200V, undervoltage; $240 \mathrm{~V}$, overvoltage) and the AVQR is under the same load condition. The supply voltage disturbances are given by a programmable source, whose output is a periodical variation as in $200 \mathrm{~V} \rightarrow 220 \mathrm{~V} \rightarrow 240 \mathrm{~V} \rightarrow 220 \mathrm{~V} \rightarrow 200 \mathrm{~V} \ldots \ldots$ and the duration of each cycle is 2s. In Fig. 18, the supply voltage disturbances are $200 \mathrm{~V}$ undervoltages in the period of UV, a $220 \mathrm{~V}$ rated voltage in the period of VS, and $240 \mathrm{~V}$ overvoltages in the period of ST. It can be seen from Fig. 18 that, when the supply voltage disturbances are overvoltages, the dc-link voltages are pumping-up. Once the dc-link voltage reaches the threshold value, the phase shift control algorithm is applied to the AVQR automatically instead of the in-phase compensation method and the dc-link voltage does not continuously rise but is gradually regulated at a constant level. The compensation voltage starts rising in a short dynamic phase shift process and reaches the steady-state at the end of the dynamic process. When supply voltage disturbances return to the rated value, the dc-link voltages gradually decline. The compensation voltages decline correspondingly and the AVQR applies the in-phase compensation method again automatically. During this whole process, the magnitudes of the output voltages are keep constant, without a sudden phase-jump. This indicates that the performance of the AVQR is excellent. It is significant that this part of the experiment is also seldom found in other studies [10]-[13], [17]-[20].

For the convenience of detailed observation, Figs. 19 22 are partial enlarged views of Fig. 18. Fig. 19 is an enlarged view of period ST; Fig. 20 is an enlarged view of period SP in Fig. 19; Fig. 21 is an enlarged view at around instant $P$ in Fig. 20; and an enlarged view at around instant $\mathrm{T}$ is shown in Fig. 22.

Comparing Figs. 14 15, which show the load-disturbance with the overvoltage condition, and Figs.19 20 which show the overvoltage supply disturbance with the load applying condition, it can be seen that the three part variations of the dc-link voltage and the compensation voltage are similar. In the in-phase compensation part, the AVQR absorbs energy, which results in a nearly linear rise of the dc-link voltage. Once the dc-link voltage reaches the threshold value, the AVQR gradually enters the phase shift compensation state. In the dynamic phase shift part, the rising rate of the dc-link voltage decreases, and the dc-link voltage is maintained at a constant level. In the steady-state phase shift compensation part, the dc-link voltage and the compensation voltage are kept stable. It can be seen from the enlarged view in Fig. 21 that, after the dynamic phase shift process, the dc-link voltage is kept constant and that there is a certain phase difference between the supply voltage and the output voltage. Fig. 22 shows the waveforms of the dc-link voltage and the compensation voltage, when the supply voltage returns to the rated value. The variations of these waveforms are the inverse of the overvoltage case.

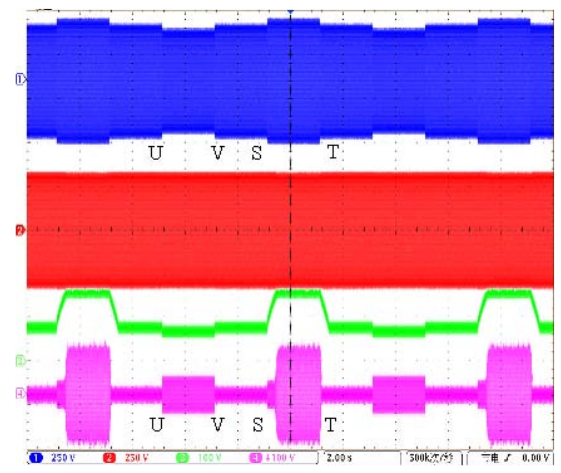

Fig. 18. Outline of the supply-disturbance experiment. (CH1: supply voltage, 250V/div; CH2: output voltage, 250V/div; CH3: dc-link voltage, 100V/div; CH4: compensation voltage, 100V/div; time axes: 2s/div).

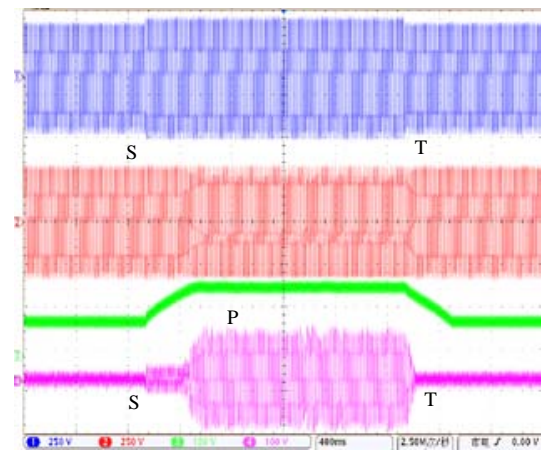

Fig.19. Enlarged view of period ST. (CH1: supply voltage, 250V/div; CH2: output voltage, 250V/div; CH3: dc-link voltage, $100 \mathrm{~V} / \mathrm{div}$; CH4: compensation voltage, $100 \mathrm{~V} / \mathrm{div}$; time axes: 400ms/div).

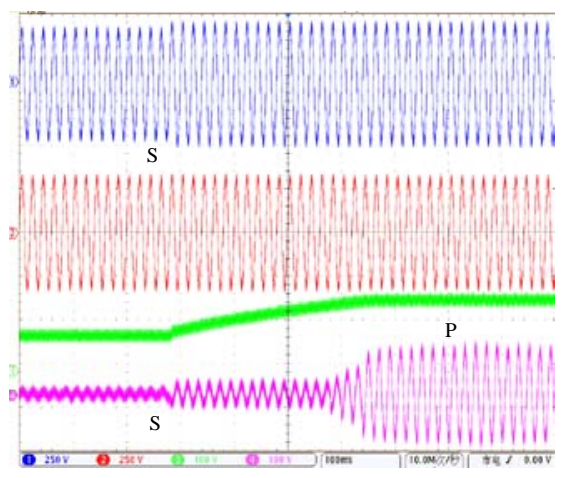

Fig. 20. Enlarged view of period SP. (CH1: supply voltage, 250V/div; CH2: output voltage, 250V/div; CH3: dc-link voltage, 100V/div; CH4: compensation voltage, 100V/div; time axes : $100 \mathrm{~ms} /$ div). 
The enlarged and detailed figures above indicate that the effectiveness of restraining both the supply voltage disturbances and the dc-link voltage pumping-up are excellent. They also show that the progressive phase shift process of the output voltage is stable and that the magnitude of the output voltage is kept at the rated value all the times.

\section{Three-phase AVQR experiment}

The three single-phase configurations shown in Fig. 10 can be further combined into a three-phase four-wire AVQR system whose topology is shown in Fig. 23. With the same load, Table III lists the steady-state test results of both the three-phase output voltages and its phase differences during undervoltage and overvoltage conditions, respectively. The results were obtained with a Power Quality Analyzer HIOKI3197 (made in Japan). It can be seen from Table III that the voltage stability of the AVQR is excellent. The output voltage is almost always maintained at the rated value of $220 \mathrm{~V}$, even when the supply voltage is experiencing undervoltage or overvoltage conditions. Especially, in the overvoltage condition, the magnitude of the three-phase output voltages is maintained at the rated value and the phase differences in the three-phase output voltages are nearly $120^{\circ}$ symmetrical. The three-phase AVQR experimental results verify the effectiveness of the phase shift control algorithm for the three-phase AVQR.

With the proposed control method, the AVQR can fully satisfy the requirements for the automatic regulation of the compensation voltage and the phase shift angle with random fluctuations of the supply voltage and the load conditions. This makes the AVQR capable of operating effectively and safely under any supply and load conditions to ensure the voltage quality for sensitive customers. This merit is verified by developing two AVQRs, with ratings of three-phase $50 \mathrm{kVA}$ and $100 \mathrm{kVA}$, based on the proposed control method.

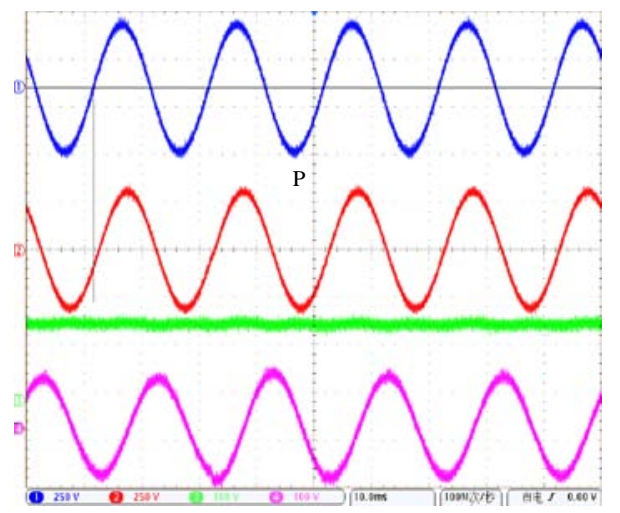

Fig. 21. Enlarged view around instant P. (CH1: supply voltage, 250V/div; CH2: output voltage, 250V/div; CH3: dc-link voltage, 100V/div; CH4: compensation voltage, 100V/div; time axes: 10ms/div).

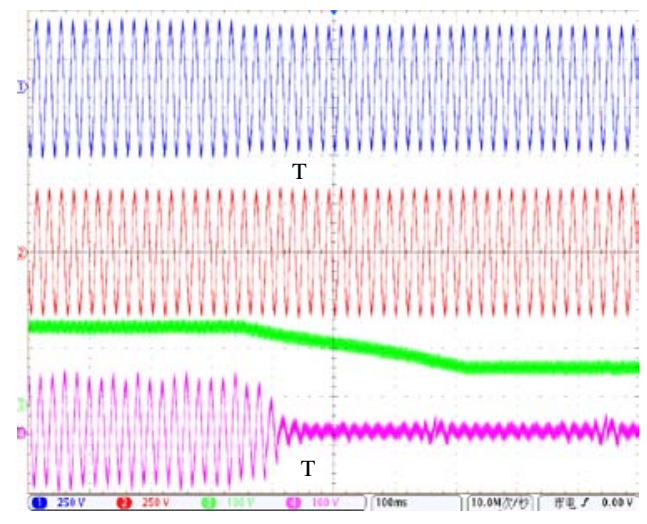

Fig. 22. Enlarged view around instant T. (CH1: supply voltage, 250V/div; CH2: output voltage, 250V/div; CH3: dc-link voltage, 100V/div; CH4: compensation voltage, 100V/div; time axes: $100 \mathrm{~ms} /$ div).

TABLE III

THREE-PHASE EXPERIMENTAL RESUlTS

\begin{tabular}{ccccccc}
\hline & \multicolumn{3}{c}{ Voltage $(\mathrm{rms}, \mathrm{V})$} & \multicolumn{4}{c}{ Phase difference $\left(^{\circ}\right)$} \\
Phase & $\mathrm{A}$ & $\mathrm{B}$ & $\mathrm{C}$ & $\mathrm{A}$ & $\mathrm{B}$ & $\mathrm{C}$ \\
$\begin{array}{c}\text { Supply voltage } \\
\text { (undervoltage) }\end{array}$ & 184.4 & 189.2 & 189.1 & 0.0 & -120.0 & 120.5 \\
Output voltage & 219.2 & 219.9 & 220.0 & 0.0 & -119.9 & 120.1 \\
\hline & \multicolumn{2}{c}{ Voltage (rms, V) } & Phase & difference $\left(^{\circ}\right)$ \\
Phase & $\mathrm{A}$ & $\mathrm{B}$ & $\mathrm{C}$ & $\mathrm{A}$ & $\mathrm{B}$ & $\mathrm{C}$ \\
$\begin{array}{c}\text { Supply voltage } \\
\text { (overvoltage) }\end{array}$ & 250.5 & 258.0 & 256.1 & 0.0 & -119.6 & 120.7 \\
Output voltage & 220.8 & 219.9 & 220.1 & 0.0 & -119.9 & 120.0 \\
\hline
\end{tabular}

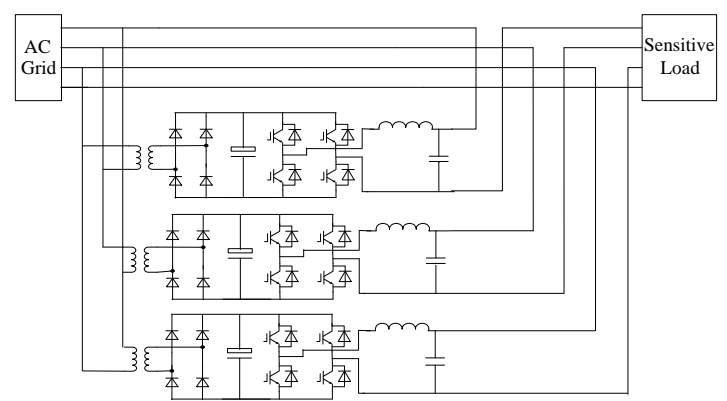

Fig. 23. Topology of a three-phase AVQR with isolation transformer.

\section{CONCLUSIONS}

The pumping-up phenomenon of the dc-link voltage in an AVQR under an overvoltage is analyzed in this paper. Then, a closed-loop control of the dc-link voltage is proposed, and the realization and application of the algorithm to suppress overvoltages are discussed and validated experimentally.

(1) The relationship between the supply overvoltage ratio, the load power factor, the phase shift angle and the compensation voltage is presented to guide in the design of 
both the main circuit and the control system.

(2) The realization of a phase shift control algorithm for both single-phase and three-phase systems is presented. The proposed algorithm can regulate the compensation voltage and the phase shift angle according to the operating condition of the power supply and the load automatically. It can keep the output voltage at a rated value, restrain the pumping-up of the dc-link voltage, ensure the safe operation of the AVQR, and realize a progressive phase shift of the output voltage without introducing a phase-jump.

(3) The effectiveness of the theoretical analysis and the proposed method is verified by simulation and experimental results for a single-phase $5 \mathrm{kVA}$ and a three-phase $15 \mathrm{kVA}$ prototype. Furthermore, two AVQRs with a rating of three-phase 50kVA and 100kVA are developed to demonstrate the practicality of the proposed algorithm.

\section{ACKNOWLEDGMENT}

This paper and its related research work were supported by the national Natural Science Foundation of China (NSFC) (project no. 50877065)

\section{REFERENCES}

[1] J. Arrillaga, M. H. J. Bollen, and N. R. Watson, "Power quality following deregulation," Proceedings of the IEEE, Vol. 88, No. 2, pp. 246-261, Feb. 2000.

[2] C. D. C. Teixeira, "Power quality solutions for low and medium voltage critical loads," in Proceeding of IEEE/PES Transmission and Distribution Conference and Exposition, pp. 326-331, 2004.

[3] J. G. Nielsen and F. Blaabjerg, "A detailed comparison of system topologies for dynamic voltage restorers," IEEE Trans. Ind. Appl., Vol. 41, No. 5, pp. 1272-1280, Sep./Oct. 2005.

[4] J. Shi, Y. Tang, K. Yang, L. Chen, L. Ren, J. Li, and Shijie Cheng, "SMES based dynamic voltage restorer for voltage fluctuations compensation," IEEE Trans. Applied Supercond., Vol. 20, No. 3, pp. 1360-1364, Jun. 2010.

[5] E. Babaei, M. F. Kangarlu, and M. Sabahi, "Mitigation of voltage disturbances using dynamic voltage restorer based on direct converters,” IEEE Trans. Power Del., Vol. 25, No. 4, pp. 2676-2683, Oct. 2010.

[6] F. A. L. Jowder, "Design and analysis of dynamic voltage restorer for deep voltage sag and harmonic compensation," IET Gener. Transm. Distrib., Vol.3, No. 6, pp. 547-560, June 2009.

[7] A. Prasai and D. M. Divan, "Zero-energy sag corrector with reduced device count,” IEEE Trans. Power Electron., Vol. 24, No. 6, pp. 1646-1653, Jun. 2009.

[8] T. X. Wang and S. S. Choi, "Enhancement of voltage quality in isolated power systems,” IEEE Trans. Power Del., Vol. 22, No. 2, pp. 1160-1168, Apr. 2007.
[9] IEEE Recommended Practice for Monitoring Electric Power Quality, IEEE Standard 1159-2009, Jun. 2009.

[10] S. S. Choi, J. D. Li, and D. M. Vilathgamuwa, “A generalized voltage compensation strategy for mitigating the impacts of voltage sags/swells," IEEE Trans. Power Del., Vol. 20, No. 3, pp. 2289-2297, Jul. 2005.

[11] S. S. Choi, B. H. Li, D. and M.Vilathgamuwa, "Dynamic voltage restoration with minimum energy injection,” IEEE Trans. Power Syst., Vol. 15, No.1, pp. 51-57, Feb. 2000.

[12] D. M. Vilathgamuwa, A. A. D. Ranjith Perera, and S. S. Choi, "Voltage sag compensation with energy optimized dynamic voltage restorer,” IEEE Trans. Power Del., Vol. 18, No. 3, pp. 928-936, Jul. 2003.

[13] T. X. Wang, S. S. Choi, and E. K. K. Sng, "Series compensation method to mitigate harmonics and voltage sags and swells," IET Gener. Transm. Distrib., Vol. 1, No. 1, pp. 96-103, Jan. 2007.

[14] C. Meyer, R. W. De Doncker, Y. W. Li, and F. Blaabjerg, "Optimized control strategy for a medium-voltage DVR-theoretical investigations and experimental results,” IEEE Trans. Power Electron., Vol. 23, No. 6, pp. 2746- 2754, Nov. 2008.

[15] J. D. Li, S. S. Choi, and D. M. Vilathgamuwa, "Impact of voltage phase jump on loads and its mitigation," in Proceeding of 4th International Power Electronics and Motion Control Conference, Vol. 3, pp. 1762-1766, 2004.

[16] J. D. Li, S. S. Choi, and D. M. Vilathgamuwa, "Impacts of voltage phase shift on motors loads and series custom power devices including converter thermal effects," IEEE Trans. Power Del., Vol. 19, No. 4, pp. 1941-1950, Oct. 2004.

[17] M. T. Tsai, “Analysis and design of a cost-effective series connected AC voltage regulator,” IEE Proc.-Electr. Power Appl., Vol. 151, No. 1, pp. 107-115, Jan. 2004.

[18] M. T. Tsai, "Design of a compact series-connected AC voltage regulator with an improved control algorithm," IEEE Trans. Ind. Electron., Vol. 51, No. 4, pp. 933-936, Aug. 2004.

[19] G. J. Li, X. P. Zhang, S. S. Choi, T. T. Lie, and Y. Z. Sun, "Control strategy for dynamic voltage restorers to achieve minimum power injection without introducing sudden phase shift," IET Gener. Transm. Distrib., Vol. 1, No. 5, pp. 847-853, Sep. 2007.

[20] C.-S. Lam, M.-C. Wong, and Y.-D. Han, "Voltage swell and overvoltage compensation with unidirectional power flow controlled dynamic voltage restorer," IEEE Trans. Power Del., Vol. 23, No. 4, pp. 2513-2521, Oct. 2008.

[21] G. Xiao, Z. Hu, C. Nan, and Z. Wang, "DC-Link voltage pumping-up analysis and phase shift control for a series active voltage regulator," in Proceeding of 37th IEEE Power Electronics Specialists Conference, pp. 1-5, 2006.

[22] J. Zhang, G. Xiao, Z. Nan, Z. Hu, B. Chen, and Z. Wang, "A research on the application of phase shift control for active voltage quality regulator," in Proceeding of 38th IEEE Power Electronics Specialists Conference, pp. 1132-1137, 2007. 


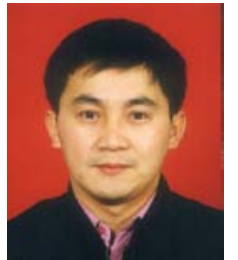

Guochun Xiao was born in Sichuan Province, China, in 1965. He received his B.S., M.S. and Ph.D. from the School of Electrical Engineering, Xi'an Jiaotong University, Xi'an, China, in 1987, 1990 and 2002, respectively. From 1990 to 1998, he was an Engineer at the Xi'an Electric Furnace Research Institute. He is now an Associate Professor at Xi'an Jiaotong University. His current research interests include power conversion systems, harmonics suppression, reactive power compensation, and active power filters.

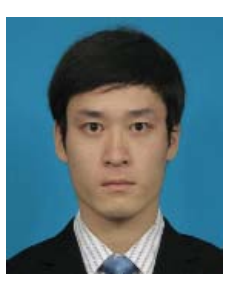

Guofei Teng was born in Shaanxi Province, China, in 1984. He received his B.S and M.S. from the School of Electrical Engineering, Xi'an Jiaotong University, Xi'an, China, in 2007 and 2010, respectively. He is currently working toward his Ph.D. at the Power Electronics and Renewable Energy Research Center, Xi'an Jiaotong University. His current research interests include power quality control and the control of photovoltaic inverters.

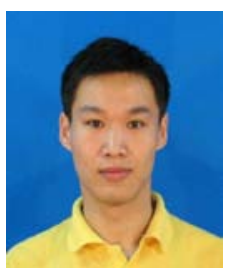

Beihai Chen was born in Anhui Province, China, in 1983. He received his B.S. and M.S. from the School of Electrical Engineering, Xi'an Jiaotong University, China, in 2006 and 2009, respectively. From 2006 to 2009, he was with the Power Electronics and Renewable Energy Research Center, Xi'an Jiaotong University, where he was a graduate student. Currently, he is working for $\mathrm{XJ}$ Corporation, as a Product Development Engineer. His current research interests include power electronics and power converters.

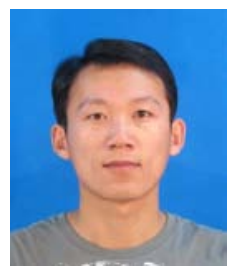

Jixu Zhang was born in Henan Province, China, in 1983. He received his B.S. in Electrical Engineering from Jilin University, Changchun, China, in 2005, and his M.S. from the School of Electrical Engineering, Xi'an Jiaotong University, Xi'an, China, in 2008. From 2005 to 2008, he was with the Power Electronics and Renewable Energy Research Center, Xi'an Jiaotong University, where he was a graduate student. Currently, he is working at the Luoyang Institute of Electro-Optic Equipment, AVIC, as a Product Development Engineer. His current research interests are in the area of compute science. 